\title{
Synchrotron Photoemission Study of Ferromagnetic (Zn,Co)O Films
}

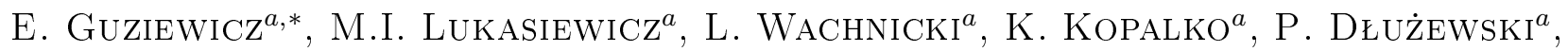 \\ R. JAKIELA ${ }^{a}$ AND M. GODLEWSKI ${ }^{a, b}$ \\ ${ }^{a}$ Institute of Physics, Polish Academy of Sciences, al. Lotników 32/46, 02-668 Warsaw, Poland \\ ${ }^{b}$ Department of Mathematics and Natural Sciences, College of Science, Cardinal S. Wyszyński University
}

01-815 Warsaw, Poland

\begin{abstract}
The electronic structure of ferromagnetic $(\mathrm{Zn}, \mathrm{Co}) \mathrm{O}$ films was investigated by resonant photoemission across the Co $3 p \rightarrow$ Co $3 d$ photoionization threshold, e.g. using photon energy between $50 \mathrm{eV}$ and $66 \mathrm{eV}$. The films were grown by atomic layer deposition at temperature between $160^{\circ} \mathrm{C}$ and $300{ }^{\circ} \mathrm{C}$ and they differed in distribution and content of cobalt. The maximum of the Fano resonance was observed at photon energy $63 \mathrm{eV}$, whereas the minimum at $58 \mathrm{eV}$. The difference between energy distribution curves taken at $63 \mathrm{eV}$ and the one taken at $58 \mathrm{eV}$ was calculated for a series of samples. It shows that the Co $3 d$ contribution to the valence band electronic structure of ferromagnetic ( $\mathrm{Zn}, \mathrm{Co}) \mathrm{O}$ films differs significantly from that of the films which show the paramagnetic response.
\end{abstract}

PACS: 73.20.At, 75.50.Pp, 81.05.Dz, 81.15.Gh

\section{Introduction}

In the last decade $(\mathrm{Zn}, \mathrm{Co}) \mathrm{O}$ focus a lot of scientific attention and became one of the most investigated materials for spintronic applications. These intensive studies started at the beginning of this century and were related to the prediction of room temperature ferromagnetism for transition metal doped zinc oxide and gallium nitride [1]. Indeed, in 2001 room temperature ferromagnetism was experimentally observed in the $(\mathrm{Zn}, \mathrm{Co}) \mathrm{O}$ system [2]. Despite the first report has been immediately followed by many others, it was soon realized that the origin of room temperature ferromagnetism in $(\mathrm{Zn}, \mathrm{Co}) \mathrm{O}$ is not clear and agreement between theory and experiment is only apparent. Ferromagnetic ordering in $(\mathrm{Zn}, \mathrm{Co}) \mathrm{O}$ has been related to defects in the $\mathrm{ZnO}$ lattice [3], formation of foreign phases [4], uncompensated spins at the $(\mathrm{Zn}, \mathrm{Co}) \mathrm{O}$ surface or metal accumulation layers [5].

In our previous studies on the $(\mathrm{Zn}, \mathrm{Co}) \mathrm{O}$ system [6] we showed that atomic layer deposition (ALD) can lead both to uniform and nonuniform ( $\mathrm{Zn}, \mathrm{Co}$ )O films depending on such process parameters as temperature, doses of precursors, and purging times. We found that the paramagnetic response was characteristic of uniform $(\mathrm{Zn}, \mathrm{Co}) \mathrm{O}$ films, while nonuniformity of cobalt distribution was prerequisite for the ferromagnetic response.

In the present work we investigate the electronic band structure of ferromagnetic $(\mathrm{Zn}, \mathrm{Co}) \mathrm{O}$ films in order to get insight into the Co $3 d$ contribution to the valence

\footnotetext{
* corresponding author; e-mail: guzel@ifpan.edu.pl
}

band in a ferromagnetic system. We performed the resonant photoemission (RESPES) experiment across the Co $3 p \rightarrow$ Co $3 d$ photoionization threshold on ferromagnetic ( $\mathrm{Zn}, \mathrm{Co}) \mathrm{O}$ samples and compared these results with the previous ones that were collected for paramagnetic films [7].

\section{Experimental}

The investigated ( $\mathrm{Zn}, \mathrm{Co}) \mathrm{O}$ films were grown on silicon substrates by the ALD method using the same zinc, cobalt and water precursors (, DMZn, Co(acac) $)_{2}$ and water, respectively). The growth method as well as growth conditions are described elsewhere [6, 8]. Ferromagnetic ( $\mathrm{Zn}, \mathrm{Co}) \mathrm{O}$ films were obtained for growth temperature between $200^{\circ} \mathrm{C}$ and $300^{\circ} \mathrm{C}$, e.g. slightly higher than temperature used for paramagnetic films $\left(160^{\circ} \mathrm{C}\right)$. Different parameters of the ALD process (precursors' doses, purging time and proportion between zinc and cobalt precursors' pulses) resulted in different Co content, which varied between $2 \%$ and $19 \%$ as obtained from the secondary ion mass spectroscopy (SIMS). The microstructure of ferromagnetic films and chemical analysis were carried out on the high resolution transmission electron microscope (HRTEM) Titan 80-300 equipped with the energy dispersive X-ray spectrometer (EDX).

The electronic structure of the $(\mathrm{Zn}, \mathrm{Co}) \mathrm{O}$ films was studied by photoemission spectroscopy in the binding energy (BE) range between the Fermi level and $14 \mathrm{eV}$ below. This $\mathrm{BE}$ region covers the $(\mathrm{Zn}, \mathrm{Co}) \mathrm{O}$ valence band together with the $\mathrm{Zn} 3 d$ core level. We used the resonant 
photoemission, which is as an effective tool for identification of the Co $3 d$ states in the valence band electronic structure of semimagnetic semicondutors with cobalt. The experiment was performed at the I4 station of the 33 beamline at the MAX III storage ring at MAXLAB, Sweden. Photoemission spectra were recorded with the overall resolution of $50 \mathrm{meV}$ at $50 \mathrm{eV}$ photon energy.

\section{Results}

The structural characterization carried out by X-ray diffraction (XRD) shows that all (Zn,Co)O films are polycrystalline. Diffraction maxima related to (10.0), (00.2) and (10.2) orientations were observed. In the HRTEM images of $(\mathrm{Zn}, \mathrm{Co}) \mathrm{O}$ films one can distinguish oval crystallites of different orientations with a diameter of 3-4 nm, which form a layer of 50-100 nm thick (Fig. 1). The qualitative analysis shows that these crystallites differ also in chemical composition. We can distinguish regions in which the value of the lattice parameter is characteristic of zinc oxide, cobalt oxide $\mathrm{CoO}$ and also the ternary compound $\mathrm{ZnCo}_{2} \mathrm{O}_{4}$. Near to the $(\mathrm{Zn}, \mathrm{Co}) \mathrm{O} / \mathrm{Si}$ interface we observe the region with the increased cobalt content. The X-ray photoelectron spectroscopy (XPS) studies show that cobalt found in the interface region is in metallic form [9].

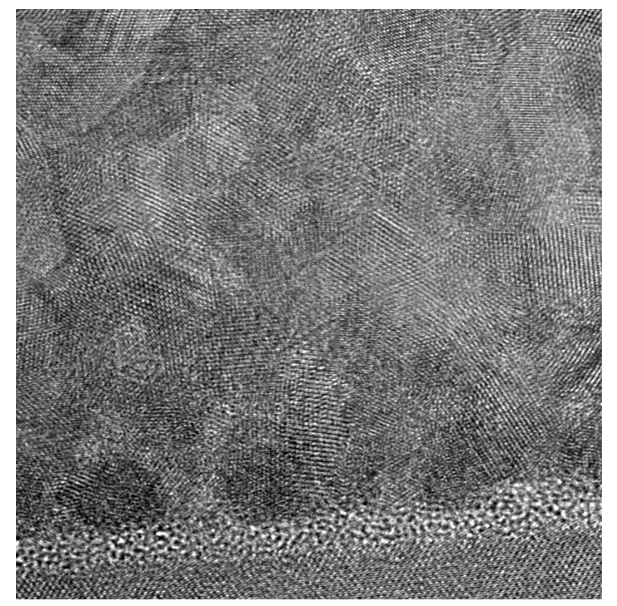

Fig. 1. HRTEM image of the F307 sample (ferromagnetic) that shows area of $40 \times 40 \mathrm{~nm}^{2}$.

We performed RESPES measurements for a series of ferromagnetic (Zn,Co)O samples. Resonant photoemission is based on the Fano effect [10]. This phenomenon occurs in photoemission when the energy of incident photons is close to the energy difference between a fully occupied core level and a partially occupied electron shell like $3 d$ shell of transition metals, $4 f$ shell of rare earth or $5 f$ shell of actinides. The resonant photoemission is a result of interference between two possible photoemission paths (classical and resonant one), that in the case of cobalt, can be described as follows:

$$
\text { Co } 3 \mathrm{p}^{6} 3 \mathrm{~d}^{7}+\mathrm{h} \nu \rightarrow\left[\text { Co } 3 \mathrm{p}^{5} 3 \mathrm{~d}^{8}\right]^{*} \rightarrow \text { Co } 3 \mathrm{p}^{6} 3 \mathrm{~d}^{6}+\mathrm{e},
$$

$$
\text { Co } 3 \mathrm{p}^{6} 3 \mathrm{~d}^{7}+\mathrm{h} \nu \rightarrow \text { Co } 3 \mathrm{p}^{6} 3 \mathrm{~d}^{6}+\mathrm{e} .
$$

As a result, we observe an increase of the Co $3 d$ related features in photoemission spectra when photon energy is tuned to the Co $3 p \rightarrow$ Co $3 d$ photoionization threshold. The maximum of the Fano resonance is preceded by the minimum which is achieved for photon energy where classical and resonant photoemission processes quench each other. Therefore the photoemission energy distribution curve (EDC) spectrum taken at the minimum of the resonance does not show any contribution from the Co $3 d$ shell and thus the difference spectra $\Delta \mathrm{EDC}=\mathrm{EDC}_{\mathrm{rez}}-\mathrm{EDC}_{\text {arez }}$ can be regarded as photoemission response from only the Co $3 d$ electron shell.

The EDC spectra were taken for photon energies between $55 \mathrm{eV}$ and $66 \mathrm{eV}$, i.e. across the Co $3 p \rightarrow$ Co $3 d$ photoionization threshold. For all investigated films the maximum of the Fano resonance was found at photon energy of $63 \mathrm{eV}$, whereas the minimum - at $58 \mathrm{eV}$. As all the samples were polycrystalline, the changes observed in the EDC spectra taken with different photon energies are not influenced by the matrix element effects and thus can be interpreted in terms of resonant photoemission. The EDC spectra taken at $h \nu=58 \mathrm{eV}$ (the Fano antiresonance) are similar to those previously reported for the $\mathrm{ZnO}$ single crystal [11].

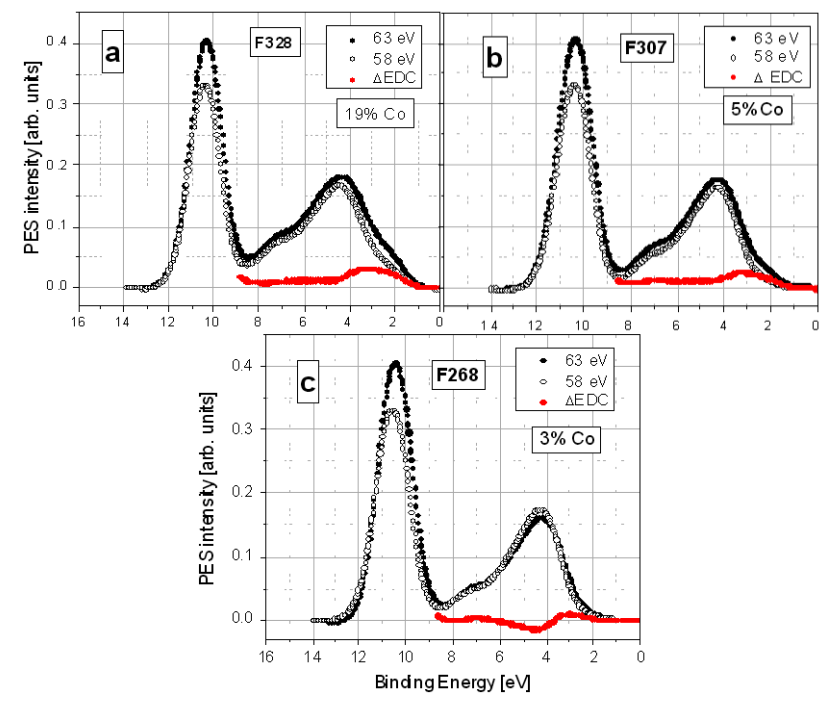

Fig. 2. Energy distribution curves taken at the maximum ( $63 \mathrm{eV}$, black solid circles) at the Fano resonance measured for ferromagnetic ( $\mathrm{Zn}, \mathrm{Co}) \mathrm{O}$ samples.

In Fig. 2 we present representative results of RESPES taken for ferromagnetic ( $\mathrm{ZnCo}) \mathrm{O}$ samples. Resonant (black solid circles), antiresonant (black open circles) and difference $\triangle \mathrm{EDC}$ spectra (red solid circles) for three of the investigated ferromagnetic $(\mathrm{Zn}, \mathrm{Co}) \mathrm{O}$ layers are presented for three $(\mathrm{Zn}, \mathrm{Co}) \mathrm{O}$ layers that differ with the cobalt content. We observed that the intensity of the resonant enhancement from the Co $3 d$ shell does not scale with the cobalt content. It was found that the 
contribution of the Co $3 d$ states to the valence band electronic structure is not the same for all investigated films, although intensities of $\triangle \mathrm{EDC}$ spectra is similar even if cobalt content is significantly different. For example, the intensity of $\triangle \mathrm{EDC}$ for sample with $19 \%$ of cobalt (Fig. 2a) is only slightly higher than for sample with $5 \%$ of cobalt (Fig. $2 \mathrm{~b}$ ). For all investigated films we find the Co $3 d$ contribution to the valence band between $2 \mathrm{eV}$ and $4 \mathrm{eV}$ below the Fermi level with the maximum at $3 \mathrm{eV}$. We do not notice a satellite structure at $7 \mathrm{eV}$ below the Fermi level, which was observed for paramagnetic $(\mathrm{Zn}, \mathrm{Co}) \mathrm{O}$ films [7]. For some samples we notice redistribution of $s-p$ valence band states at $4.5 \mathrm{eV}$ below the Fermi level (Fig. 2c) which results in negative value of the $\triangle \mathrm{EDC}$ spectrum between $4 \mathrm{eV}$ and $6 \mathrm{eV}$ below the Fermi level.

\section{Conclusions}

We investigated a set of ferromagnetic ( $\mathrm{Zn}, \mathrm{Co}) \mathrm{O}$ films. Resonant photoemission studies show that the contribution of the Co $3 d$ electron states to the valence band electronic structure is observed between $2 \mathrm{eV}$ and $4 \mathrm{eV}$ below the Fermi level with the maximum at $3 \mathrm{eV}$. We do not observe any enhancement of the resonant photoemission signal that was observed for paramagnetic $(\mathrm{Zn}, \mathrm{Co}) \mathrm{O}$ films and that was ascribed to the satellite structure $[12,13]$.

\section{Acknowledgments}

The research was partially supported by the funding from the EC FP7 Programme (FP7/2007-2013) under grant agreement no. 226716 and by the EU within the European Regional Development Fund, through grant Innovative Economy (POIG.01.01.02-00-008/08).

\section{References}

[1] T. Dietl, H. Ohno, F. Matsukura, J. Cibert, D. Ferrand, Science 287, 1019 (2000).
[2] K. Ueda, H. Tabata, T. Kawaii, Appl. Phys. Lett. 79, 988 (2001).

[3] J.M.D. Coey, K. Wongsaprom, J. Alaria, M. Venkatesan, J. Phys. D, Appl. Phys. 41, 134012 (2008).

[4] A.J. Behan, A. Mokhtari, H.J. Blythe, D. Score, X.-H. Xu, J.R. Neal, A.M. Fox, G.A. Gehring, Phys. Rev. Lett. 100, 047206 (2008).

[5] J.H. Park, M.G. Kim, H.M. Jang, S. Ryu, Y.M. Kim, Appl. Phys. Lett. 84, 1338 (2004).

[6] M.I. Łukasiewicz, B. Witkowski, M. Godlewski, E. Guziewicz, M. Sawicki, W. Paszkowicz, E. Łusakowska, R. Jakieła, T. Krajewski, I.A. Kowalik, B.J. Kowalski, Acta Phys. Pol. A 116, 921 (2009).

[7] E. Guziewicz, M.I. Lukasiewicz, L. Wachnicki, K. Kopalko, A. Kovács, R.E. Dunin-Borkowski, B.S. Witkowski, B.J. Kowalski, J. Sadowski, M. Sawicki, R. Jakiela, M. Godlewski, Radiat. Phys. Chem. 80, 1046 (2011).

[8] M.I. Łukasiewicz, B. Witkowski, M. Godlewski, E. Guziewicz, M. Sawicki, W. Paszkowicz, R. Jakieła, T.A. Krajewski, G. Łuka, Phys. Status Solidi B 247, 1666 (2010).

[9] M. Godlewski, E. Guziewicz, M.I. Łukasiewicz, I.A. Kowalik, M. Sawicki, B.S. Witkowski, R. Jakieła, W. Lisowski, J.W. Sobczak, M. Krawczyk, Phys. Status Solidi B 248, 1596 (2011).

[10] U. Fano, Phys. Rev. 124, 1866 (1961)

[11] R.T. Girard, O. Tjernberg, G. Chiaia, S. Soderholm, U.O. Karlson, C. Wigren, H. Nylen, I. Linden, Surf. Sci. 373, 409 (1997).

[12] M. Kobayashi, Y. Ishida, J.I. Hwang, T. Mizokawa, A. Fuijmori, K. Mamiya, J. Okamoto, Y. Takeda, T. Okane, Y. Saitoh, Y. Muramatsu, A. Tanaka, H. Saeki, H. Tabata, T. Kawai, Phys. Rev. B 72, 201201(R) (2005).

[13] S.C. Wi, J.-S. Kang, J.H. Kim, S.-B. Cho, B.J. Kim, S. Yoon, B.J. Suh, S.W. Han, K.H. Kim, K.J. Kim, B.S. Kim, H.J. Song, H.J. Shin, J.H. Shim, B.I. Min, Appl. Phys. Lett. 84, 4233 (2004). 